PROCEEDINGS OF THE

AMERICAN MATHEMATICAL SOCIETY

Volume 134, Number 11, November 2006, Pages 3267-3274

S 0002-9939(06)08447-4

Article electronically published on June 6, 2006

\title{
A WEYL TYPE FORMULA FOR FOURIER SPECTRA AND FRAMES
}

\author{
ALEX IOSEVICH AND MIHAIL N. KOLOUNTZAKIS
}

(Communicated by Michael T. Lacey)

\begin{abstract}
We prove qualitative and quantitative results concerning the asymptotic density in dilates of centered convex bodies of the frequency vectors of orthogonal exponential bases and frames associated to bounded domains in Euclidean space.
\end{abstract}

\section{INTRODUCTION}

Let $\Omega \subset \mathbb{R}^{d}$ be a bounded domain. We say that $\Omega$ is spectral if $L^{2}(\Omega)$ possesses an orthogonal basis of the form $E_{\Lambda}=\left\{e^{2 \pi i x \cdot \lambda}\right\}_{\lambda \in \Lambda}$, where $\Lambda$ is a subset of $\mathbb{R}^{d}$. We shall refer to $\Lambda$ as a spectrum of $\Omega$. The set $\Lambda$ is easily seen to have a separation property: $|\lambda-\mu| \geq \epsilon$ for all $\lambda, \mu \in \Lambda, \lambda \neq \mu$, and is therefore a discrete set.

A systematic study of such spectra was initiated by Fuglede in 11. This problem has received much recent attention.

Let

$$
D_{R}^{+}(\Omega)=\max _{x \in \mathbb{R}^{d}} \#\left\{\Lambda \cap Q_{R}(x)\right\}
$$

and

$$
D_{R}^{-}(\Omega)=\min _{x \in \mathbb{R}^{d}} \#\left\{\Lambda \cap Q_{R}(x)\right\}
$$

where $Q_{R}(x)$ denotes the cube of side-length $2 R$ centered at $x$. Landau [7] proved that

$$
\limsup _{R \rightarrow \infty} \frac{D_{R}^{+}}{(2 R)^{d}}=|\Omega|,
$$

and the same equality holds for $D_{R}^{-}$.

If $E_{\Lambda}$ is only a frame for $L^{2}(\Omega)$, in the sense that there exist positive constants $A$ and $B$ such that

$$
A\|g\|_{L^{2}(\Omega)}^{2} \leq \sum_{A}|\hat{g}(\lambda)|^{2} \leq B\|g\|_{L^{2}(\Omega)}^{2},
$$

Received by the editors May 24, 2005.

2000 Mathematics Subject Classification. Primary 42B05.

The research of the first author was partially supported by NSF Grant DMS02-45369. The research of the second author was partially supported by European Commission IHP Network HARP (Harmonic Analysis and Related Problems), Contract Number: HPRN-CT-2001-00273 HARP. 
where $\hat{g}$ denotes the Fourier Transform of $g$, then one can only conclude that

$$
\limsup _{R \rightarrow \infty} \frac{D_{R}^{-}}{(2 R)^{d}} \geq|\Omega| \text {. }
$$

Observe that $E_{\Lambda}$ is an orthogonal basis if and only if (2) holds with $A=B=1$.

In [4, the authors proved that if $\partial \Omega$ has Minkowski dimension $\alpha<d$, with $\alpha$-dimensional upper Minkowski content denoted by $|\partial \Omega|_{\alpha},(2)$ holds, and

$$
R \geq C\left(\frac{B|\partial \Omega|_{\alpha}}{A|\Omega|}\right)^{\frac{1}{d-\alpha}}
$$

for some $C>0$, then for every $x \in \mathbb{R}^{d}, Q_{R}(x)$ contains at least one element of $\Lambda$.

In $\oint 3$ we show an alternative estimate for the minimum $R$ such that every cube of side-length $R$ necessarily contains a point of an $(A, B)$-frame for the domain. For the quite general case of planar domains which are simple polygons, we show that this estimate is always as good as (4) and often much better.

The main purpose of this paper is to prove a Weyl type estimate (see e.g. 9, Ch. $5]$ for a description of the classical Weyl asymptotics in the context of Riemannian manifolds) for $\#\{\Lambda \cap R \cdot K\}$, where $K$ is a convex body in $\mathbb{R}^{d}$, symmetric with respect to the origin, in the case when $E_{\Lambda}$ is an orthogonal basis for $L^{2}(\Omega)$. In analogy with the classical Weyl formula we ask whether

$$
\#\left\{\lambda:\|\lambda\|_{K} \leq R\right\}=c_{\Omega, \Lambda} R^{d}+o\left(R^{d}\right),
$$

where $\|\cdot\|_{K}$ is the norm induced by $K$.

It turns out that the answer is yes, and under some additional assumptions we obtain more quantitative estimates on the error term. We shall see that our estimates imply both (11) and (4) at the same time, thus presenting a unified description of geometric properties of spectra.

Theorem 1. Let $\Omega$ be a bounded domain in $\mathbb{R}^{d}$ of positive Lebesgue measure. Let $E_{\Lambda}$ be defined as above. If $E_{\Lambda}$ is an orthogonal basis for $L^{2}(\Omega)$, then

$$
\#\left\{\lambda \in \Lambda:\|\lambda\|_{K} \leq R\right\}=|K| R^{d}|\Omega|+E(R),
$$

with

$$
E(R)=o\left(R^{d}\right) .
$$

Moreover, if the boundary of $\Omega$ has upper Minkowski dimension $\alpha<d$, then

$$
|E(R)| \leq C_{K}|\partial \Omega|_{\alpha} R^{\alpha},
$$

where $C$ is a constant depending on the domain $\Omega$ only.

If $E_{\Lambda}$ is merely a frame with constants $A$ and $B$ as in (2) above, then

$$
A|\Omega| \leq \frac{\#\left\{\lambda \in \Lambda:|| \lambda \|_{K} \leq R\right\}}{|K| R^{d}} \leq B|\Omega| .
$$

Remark 1 . We do not have an example of a domain $\Omega$ with

$$
\limsup _{R \rightarrow \infty} \frac{|E(R)|}{R^{\alpha}}>0
$$

for some $\alpha>d-1$. A family of examples with $\alpha=d-1$ is given in Example 1 below. Nevertheless, the upper Minkowski assumption allows us to obtain better results than the $o\left(R^{d}\right)$ estimate obtained without any assumption on the boundary of $\Omega$. 
Observe that (4) follows immediately from (5) and (7) in the case $\Lambda$ is a spectrum, while (11) and (3) follow from (6) and (8), respectively. To see the former, we use the fact that the proof of (5) and (7) shows that the same estimate still holds with uniform constants for a translated spectrum, i.e. $\#\left\{\lambda \in \Lambda:\|\lambda-x\|_{K} \leq R\right\}=$ $|K| R^{d}|\Omega|+E(x, R)$, with $|E(x, R)| \leq C_{K}|\partial \Omega|_{\alpha} R^{\alpha}$ with constants independent of $x$, under the assumption that the boundary of $\Omega$ has upper Minkowski dimension $\alpha<d$. It follows that if $C_{K}|\partial \Omega|_{\alpha} R^{\alpha}$ is much smaller than the main term $|K| R^{d}|\Omega|$, the set $\left\{\lambda \in \Lambda:\|\lambda-x\|_{K} \leq R\right\}$ is not empty, and (4) follows.

It is interesting to compare Theorem 11 with a model situation where $\Omega=[0,1]^{d}$, $K$ is the unit Euclidean ball of volume $\omega_{d}$, and $\Lambda=\mathbb{Z}^{d}$. In this case, it is known that

$$
\#\{\lambda \in \Lambda:|\lambda| \leq R\}=\omega_{d} R^{d}+O\left(R^{d-2}\right),
$$

if $d \geq 5$, and

$$
\#\{\lambda \in \Lambda:|\lambda| \leq R\}=\omega_{d} R^{4}+O\left(R^{2} \log (R)\right),
$$

if $d=4$. In two and three dimensions the situation is more murky. In two dimensions, Hardy's conjecture says that the remainder should be $O\left(R^{\frac{1}{2}+\epsilon}\right)$, for any $\epsilon>0$. The best known result in dimension two, due to Huxley, gives $O\left(R^{\frac{46}{73}}\right)$. In three dimensions, the best known result is due to Heath-Brown who obtained $O\left(R^{\frac{21}{16}}\right)$. See 2 and the references contained therein.

All these results are driven by the curvature of the boundary of the Euclidean ball. To see this observe that if $\Omega=[0,1]^{d}, \Lambda=\mathbb{Z}^{d}$, and $K=[-1,1]^{d}$, the best we can say is

$$
\#\left\{\lambda \in \Lambda:\|\lambda\|_{K} \leq R\right\}=2^{d} R^{d}+O\left(R^{d-1}\right),
$$

since an integer dilate of the unit cube contains $\approx R^{d-1}$ integer lattice points on its boundary.

Observe that the remainders in all these results are much better than the ones given by Theorem 1 especially in the case where $K$ is the Euclidean ball. However, the following construction shows that even when $\Omega$ is the unit cube in $\mathbb{R}^{d}$ and $K$ is the Euclidean ball, the situation becomes much worse when the integer lattice is replaced by a more complicated spectrum.

Example 1. Consider the standard lattice of cubes in $\mathbb{R}^{d}$. View each cube as a translate of $[0,1]^{d}$. We call the image of the origin under this translation the defining vertex of the cube.

Consider a sphere of radius 100 centered at the origin. Move each relevant column of cubes (cubes whose defining vertices only differ in the coordinate $x_{d}$ ) in such a way that the defining vertex of one the cubes in the column is on this sphere. Now consider a sphere radius $2^{100}$. Leave the previously moved columns alone, and move the other columns in such a way that the defining vertex of one of the cubes in each column is on this sphere. Continuing this process, we produce a family of spheres with radii $\left\{R_{i}\right\}, R_{i} \rightarrow \infty$, and a discrete set $\Lambda^{\prime}$ such that the $i$ 'th sphere contains $\approx R_{i}^{d-1}$ points of $\Lambda^{\prime}$ with constants independent of $i$.

Since $[0,1]^{d}+\Lambda^{\prime}$ is clearly a tiling, the result proved independently by Lagarias, Reeds and Wang [8] and Iosevich and Pedersen [3] (see also [5]) implies that $E_{\Lambda^{\prime}}$ is an orthogonal basis for $L^{2}(\Omega)$. It is clear that in this case the estimate (7) cannot be improved. 


\section{Proof of Theorem 1}

Suppose that $E_{\Lambda}$ is an orthogonal basis for $L^{2}(\Omega)$. It follows (see e.g. [5] or Remark 2 below) that

$$
\sum_{\lambda \in \Lambda} f(x-\lambda)=|\Omega|^{2},
$$

where $f=|\widehat{\chi \Omega}|^{2}$. Note also that $\int f=|\Omega|$.

Let $K_{t}$ denote the set $t \cdot K$, let $\Lambda_{t}$ denote the set $\Lambda \cap K_{t}$ and finally let

$$
N(t)=\# \Lambda_{t}
$$

From the orthogonality of the exponentials in $E_{\Lambda}$ it follows that for any two distinct points $\lambda, \mu \in \Lambda$ their difference is a zero of $f$, hence there is a lower bound for their distance. This separation means that $N(t) \leq C t^{d}$ and also that $N(t+R)-N(t) \leq$ $C t^{d-1} R$ if $R>0$ is a large enough constant. Roughly speaking, if a domain is not too thin, then the number of $\Lambda$-points in it is bounded above by its volume.

Remark 2. If $E_{\Lambda}$ is only a frame for the space $L^{2}(\Omega)$ with frame constants $A$ and $B$, then it follows that, for $f=\widehat{\chi \Omega}$ we have for almost all $x \in \mathbb{R}^{d}$

$$
A|\Omega|^{2} \leq \sum_{\lambda \in \Lambda} f(x-\lambda) \leq B|\Omega|^{2} .
$$

This is easily seen by applying (2) to an arbitrary exponential function. The proof below for the case of $E_{\Lambda}$ being an orthonormal basis also gives (8) with trivial modifications: the separation property does not hold for frames, however using the upper bound in (13) one easily gets that $\Lambda$ has the property that matters in the proof below, namely that the number of $\Lambda$-points in $K$-balls and shells is controlled by the volume of the region.

First we follow [6] to show that $N(t)=|K||\Omega| t^{d}+o\left(t^{d}\right)$, under no assumptions about $\partial \Omega$. For this let $1<R<T$ be large numbers and integrate (12) over the region $K_{T}$ to get

$$
\begin{aligned}
|\Omega|^{2}\left|K_{T}\right|= & \int_{K_{T}} \sum_{\lambda \in \Lambda} f(x-\lambda) d x \\
= & \int_{K_{T}} \sum_{\Lambda_{T-R}} f(x-\lambda) d x+\int_{K_{T}} \sum_{\Lambda_{T+R} \backslash \Lambda_{T-R}} f(x-\lambda) d x \\
& +\int_{K_{T}} \sum_{\Lambda \backslash \Lambda_{T+R}} f(x-\lambda) d x \\
= & \int_{\mathbb{R}^{d}} \sum_{\Lambda_{T-R}} f(x-\lambda) d x-E_{1}+E_{2}+E_{3} \\
= & N(T-R)|\Omega|-E_{1}+E_{2}+E_{3},
\end{aligned}
$$

so that

$$
N(T-R)=\left|K_{T}\right||\Omega|-\frac{E_{1}}{|\Omega|}+\frac{E_{2}}{|\Omega|}+\frac{E_{3}}{|\Omega|},
$$


where

$$
\begin{aligned}
E_{1} & =\int_{K_{T}^{c}} \sum_{\Lambda_{T-R}} f(x-\lambda) d x, \\
E_{2} & =\int_{K_{T}} \sum_{\Lambda_{T+R} \backslash \Lambda_{T-R}} f(x-\lambda) d x, \\
E_{3} & =\int_{K_{T}} \sum_{\Lambda \backslash \Lambda_{T+R}} f(x-\lambda) d x .
\end{aligned}
$$

Let $\epsilon>0$ be arbitrary and fixed, and choose $R$ so that

$$
\int_{K_{R}^{c}} f(x) d x \leq \epsilon .
$$

We have, since $T>R$,

$$
\begin{aligned}
E_{1} & \leq \epsilon N(T-R) \\
& \leq \epsilon N(T) \\
& \leq C \epsilon T^{d}
\end{aligned}
$$

and

$$
\begin{aligned}
E_{3} & =\sum_{\Lambda \backslash \Lambda_{T+R}} \int_{\lambda+K_{T}} f(x) d x \\
& \leq C T^{d} \int_{K_{R}^{c}} f(x) d x \\
& \leq C \epsilon T^{d},
\end{aligned}
$$

as each point in $K_{R}^{c}$ is contained in at most $C T^{d}$ of the sets $\lambda+K_{T}$, with $\lambda \in$ $\Lambda \backslash \Lambda_{T+R}$. For $E_{2}$ we trivially have

$$
\begin{aligned}
E_{2} & \leq(N(T+R)-N(T-R))|\Omega| \\
& =o\left(T^{d}\right), \text { as } R \text { is fixed. }
\end{aligned}
$$

Since $\epsilon$ is arbitrary and $N(T-R)=N(T)-o\left(T^{d}\right)$ as $T \rightarrow \infty$, we have proved that

$$
N(T)=|K||\Omega| T^{d}+o\left(T^{d}\right) .
$$

In other words the set $\Lambda$ has density $|\Omega|$.

Let us now assume that $\partial \Omega$ has finite $\alpha$-dimensional upper Minkowski content, with $\alpha<d$. We shall prove that

$$
\int_{K_{2 R} \backslash K_{R}} f(x) d x \leq C_{K}|\partial \Omega|_{\alpha} R^{-(d-\alpha)},
$$

where $|\partial \Omega|_{\alpha}$ denotes the Minkowski content of $\partial \Omega$.

To see (14) we may of course assume that $2^{j} \sim R$, and we then choose $N$ (independent of $j$ ) boxes $Q_{\nu}$ and $N$ vectors $h_{\nu}$, with $2^{-(j+1)} \leq\left\|h_{\nu}\right\|_{K} \leq 2^{-j}$, such that the region $\left\{y: 2^{j} \leq\|y\|_{K} \leq 2^{j+1}\right\}$ is contained in $\bigcup Q_{\nu}$ and

$$
\left|e^{2 \pi i y \cdot h_{\nu}}-1\right| \geq 1 \quad\left(y \in Q_{\nu}\right) .
$$


We now have

$$
\begin{aligned}
\int_{2^{j} \leq\|y\|_{K} \leq 2^{j+1}} f(y) d y & \leq \sum_{\nu=1}^{N} \int_{Q_{\nu}}\left|\widehat{\chi_{\Omega}}(y)\left(e^{2 \pi i y \cdot h_{\nu}}-1\right)\right|^{2} d y \\
& \leq \sum_{\nu=1}^{N} \int_{\mathbb{R}^{d}}\left|\chi_{\Omega}(x)-\chi_{\Omega}\left(x-h_{\nu}\right)\right|^{2} d x \\
& \leq \sum_{\nu=1}^{N}\left|\left\{x \in \mathbb{R}^{d}: \operatorname{dist}(x, \partial \Omega)<h_{\nu}\right\}\right| \\
& \leq C \sum_{\nu=1}^{N}|\partial \Omega|_{\alpha}\left|h_{\nu}\right|^{d-\alpha} \\
& \leq C_{K}|\partial \Omega|_{\alpha} 2^{-j(d-\alpha)} .
\end{aligned}
$$

Let $R \rightarrow \infty, \epsilon=\int_{K_{R}^{c}} f=O\left(R^{-(d-\alpha)}\right)$ and integrate (12) on $K_{R}$ to get, similar to what we did above,

$$
N(R)=|\Omega|\left|K_{R}\right|-\frac{E_{1}}{|\Omega|}+\frac{E_{2}}{|\Omega|}+\frac{E_{3}}{|\Omega|},
$$

where now

$$
\begin{aligned}
E_{1} & =\int_{K_{R}^{c}} \sum_{\Lambda_{R}} f(x-\lambda) d x \\
E_{2} & =\int_{K_{R}} \sum_{\Lambda_{2 R} \backslash \Lambda_{R}} f(x-\lambda) d x \\
E_{3} & =\int_{K_{R}} \sum_{\Lambda \backslash \Lambda_{2 R}} f(x-\lambda) d x .
\end{aligned}
$$

We have as before

$$
E_{3} \leq R^{d} \int_{K_{R}^{c}} f=O\left(R^{\alpha}\right)
$$

To bound $E_{1}$ we decompose the set $\Lambda_{R}$ in shells of width $2^{j}, j=0,1, \ldots, \log _{2} R$ :

$$
A_{j}=\Lambda \cap\left(K_{R-2^{j}} \backslash K_{R-2^{j+1}}\right),
$$

thinner near $\partial K_{R}$ and doubling in width as we move towards the origin. Using (14) we get

$$
\begin{aligned}
E_{1} & \leq \sum_{j=0}^{\log _{2} R} \# A_{j} 2^{-j(d-\alpha)} \\
& \leq C \sum_{j=0}^{\log _{2} R} R^{d-1} 2^{j} 2^{-j(d-\alpha)} \\
& \leq C R^{d-1} \sum_{j=0}^{\log _{2} R} 2^{j(1-d+\alpha)} \\
& =O\left(R^{\alpha}\right) .
\end{aligned}
$$


We bound $E_{2}$ similarly by decomposing in dyadic shells the set $\Lambda_{2 R} \backslash \Lambda_{R}$, thinner near $\partial K_{R}$ and doubling as we move out:

$$
B_{j}=\Lambda \cap\left(K_{R+2^{j+1}} \backslash K_{R+2^{j}}\right)
$$

We get

$$
\begin{aligned}
E_{2} & \leq \sum_{j=0}^{\log _{2} R} \# B_{j} 2^{-j(d-\alpha)} \\
& \leq C \sum_{j=0}^{\log _{2} R}\left(R+2^{j}\right)^{d-1} 2^{j} 2^{-j(d-\alpha)} \\
& \leq C R^{d-1} \sum_{j=0}^{\log _{2} R} 2^{j(1-d+\alpha)} \\
& =O\left(R^{\alpha}\right)
\end{aligned}
$$

We have proved

$$
N(R)=|\Omega||K| R^{d}+O\left(R^{\alpha}\right)
$$

\section{An IMPROVED UPPER BOUND FOR THE SIDE-LENGTH OF EMPTY CUBES}

In this section we show a very simple new upper bound on the size of $\Lambda$-free cubes, when $E_{\Lambda}$ is a frame for $L^{2}(\Omega)$. This bound is not affected by the roughness of $\partial \Omega$ but only by how "thick" $\Omega$ is.

Theorem 2. Suppose $\Omega \subseteq \mathbb{R}^{d}$ is measurable with positive measure and also that the point set $\Lambda \subseteq \mathbb{R}^{d}$ is such that $E_{\Lambda}$ is a frame for $L^{2}(\Omega)$ with lower and upper frame constants being $A$ and $B$, respectively. Let $R$ be such that there is a cube in $\mathbb{R}^{d}$ of side-length $R$ containing no point of $\Lambda$. Finally, assume that $\Omega$ contains a cube of side-length $\epsilon>0$. Then

$$
R \leq C_{d} \frac{B}{A} \epsilon^{-1} .
$$

Proof. Let $Q \subseteq \Omega$ be a cube of side-length $\epsilon$ contained in $\Omega$. By the definition of a frame (2) it follows that $E_{\Lambda}$ is a frame for $L^{2}(Q)$ with the same frame constants. Applying (44) for $Q$, with $\alpha=d-1$, we get the result.

The new estimate (15) can easily be seen to be much better than (4) in some cases. Take for example in $\mathbb{R}^{2}$ a perturbation of the unit square that creates a very long boundary but leaves intact a square of side $1 / 2$ inside. Also, in the quite general case of simple polygonal domains, the new estimate (15) is at least as good as (4), as the following theorem claims.

Theorem 3. Suppose $\Omega$ is a simple polygon and let $\epsilon$ be the maximum side-length of a cube contained in $\Omega$. Then, for some constant $C>0$, we have

$$
\frac{|\partial \Omega|}{|\Omega|} \geq C \epsilon^{-1}
$$

Proof. Consider the usual subdivision of the plane into squares of side $\epsilon$, translated at the points $(\epsilon \mathbb{Z})^{2}$. Let $N$ be the number of those squares intersecting $\Omega$. It follows that $N \geq|\Omega| \epsilon^{-2}$. 
By our assumption about $\epsilon$ these squares all contain some point of $\Omega^{c}$ and therefore also some point of $\partial \Omega$. Let these squares be called $Q_{i}, i=1, \ldots, N$, and let $p_{i} \in Q_{i} \cap \partial \Omega$.

Partition the set of these squares into four classes depending on the parity of the $x$ - and $y$-coordinate of their lower left corner (after multiplying these coordinates by $\left.\epsilon^{-1}\right)$. At least one of these classes contains at least $N / 4$ squares. There are therefore at least $N / 4$ points on $\partial \Omega$ with minimum distance $C \epsilon$ from each other, and these are connected to each other along $\partial \Omega$. It follows that the total length of $\partial \Omega$ is at least

$$
C \frac{N}{4} \epsilon \geq C|\Omega| \epsilon^{-1}
$$

\section{REFERENCES}

[1] B. Fuglede, Commuting self-adjoint partial differential operators and a group theoretic problem, J. Funct. Anal. 16 (1974), pp. 101-121. MR0470754 (57:10500)

[2] M. N. Huxley, Area, Lattice Points, and Exponentials Sums, London Mathematical Society Monographs New Series 13 (1996), Oxford Univ. Press. MR.1420620 (97g:11088)

[3] A. Iosevich and S. Pedersen, Spectral and tiling properties of the unit cube, Internat. Math. Reseach Notices 16 (1998), pp. 819-828. MR1643694(2000d:52015)

[4] A. Iosevich and S. Pedersen, How large are the spectral gaps?, Pacific J. Math. 192 (2000), pp. 307-314. MR1744572 (2001b:42038)

[5] M.N. Kolountzakis, Packing, tiling, orthogonality and completeness, Bull. London Math. Soc. 32 (2000), 5, pp. 589-599. MR.1767712 (2001g:52030)

[6] M.N. Kolountzakis and J.C. Lagarias, Structure of tilings of the line by a function, Duke Math. J. 82 (1996), 3, pp. 653-678. MR1387688(97d:11124)

[7] H. Landau, Necessary density conditions for sampling and interpolation of certain entire functions, Acta Math. 117 (1967), pp. 37-52. MR0222554 (36:5604)

[8] J. Lagarias, J. Reeds, and Y. Wang, Orthonormal bases of exponentials for the $n$-cube, Duke Math. J. 103 (2000), 1, pp. 25-37. MR.1758237 (2001h:11104)

[9] C. Sogge, Fourier integrals in classical analysis, Cambridge University Press, 1993. MR.1205579 (94c:35178)

[10] R. Strichartz, Fourier asymptotics of fractal measures, J. of Func. Anal. 89 (1990), pp. 154187. MR1040961 (91m:42015)

Department of Mathematics, University of Missouri, Columbia, Missouri 65211

E-mail address: iosevich@math.missouri.edu

Department of Mathematics, University of Crete, Knossos Ave., GR-714 09, Iraklio, GREECE

E-mail address: kolount@member.ams.org 